Review

\title{
Recent progress in cerium-based nanomaterials for electrochemical biosensors
}

\author{
Xinli Tian ${ }^{1}$, Zhongmin Wang ${ }^{1}$, Mengkui Ding ${ }^{1}$, Shuang Zhou ${ }^{2}$, Ruizhuo Ouyang $^{1, *}$, Yuqing Miao ${ }^{1}$ \\ ${ }^{1}$ Institute of Bismuth Science, University of Shanghai for Science and Technology, Shanghai 200093, \\ China \\ ${ }^{2}$ Cancer Institute, Tongji University School of Medicine, Shanghai 200092, China \\ *E-mail: ouyangrz@usst.edu.cn
}

doi: $10.20964 / 2020.10 .12$

Received: 19 March 2020 / Accepted: 23 April 2020 / Published: 31 August 2020

Cerium $(\mathrm{Ce})$ is a rare earth element widely used for its excellent physical and chemical properties. Cebased nanomaterials have become highly relevant for electrochemical biosensors due to their attractive catalytic activities and electrical conductivity properties. Importantly, Ce-based nanomaterial preparation significantly affects their properties for electrochemical applications. This review summarizes some common methods for Ce-based nanomaterial synthesis, including sol-gel, coprecipitation and micro-emulsion approaches, and their applications in electrochemical biosensors, such as DNA sensors, immunosensors and enzyme sensors. Also, we summarize by discussing some defects and complementary methods of Ce-based nanomaterials.

Keyword: $\mathrm{CeO}_{2}$; Ce-based materials; electrochemical biosensor; preparation method

\section{FULL TEXT}

(C) 2020 The Authors. Published by ESG (www.electrochemsci.org). This article is an open access article distributed under the terms and conditions of the Creative Commons Attribution license (http://creativecommons.org/licenses/by/4.0/). 